\section{\$34. Variational Approach to a Turbulent Swirling Pipe Flow with the Aid of Helicity}

Yoshizawa, A., Yokoi, N., Nishizima, S. (Univ. Tokyo), Itoh, S.-I. (Kyushu U.),

Itoh, K.

A turbulent swirling flow in a circular pipe is studied with the aid of a variational method. The turbulent-energy production effect, which is linked with spatial velocity gradients, expresses the energy cascade from mean to turbulent components of motion and is a cause of the loss of distinct mean-flow structures. In the turbulent-energy equation, weakening of the production effect tends to enhance the diffusion effect of turbulent energy, which is also harmful to the sustainment of such structures. An optimum state in which these two effects are reconciled with each other is sought with resort to a variational approach combined with helicity. The resulting state is expressed by the mean vorticity proportional to its curl. On this basis, the centerline axial vorticity is shown to play a central role in the occurrence of axialvelocity retardation or reversal in a turbulent swirling pipe flow.[1]

A retarded or reversed axial velocity in a swirling pipe flow is characterized by the mean velocity gradient, as was noted above. As an indicator of the strength of spatial variation or inhomogeneity of mean velocity in a region $V$, we introduce

$$
\Phi=\int_{V}\left(\frac{\partial U_{j}}{\partial x_{i}}\right)^{2} d V .
$$

A mean axial vorticity arising from a mean swirl velocity is an important quantity characterizing a turbulent swirling flow in a pipe. Its combination with a mean axial velocity results in nonvanishing mean-flow helicity. Then as a critical quantity distinguishing between swirling and nonswirling flows, we introduce a total amount of mean-flow helicity, which is defined by

$$
\Psi=\int_{V} \boldsymbol{U} \cdot \boldsymbol{\Omega} d V .
$$

Effects of helicity on the energy cascade in isotropic turbulence were examined with the aid of the eddy-damped quasi-normal Markovianized (EDQNM) approximation. The study shows that the helicity in the energy-containing range of wavenumber space hampers the cascade of energy towards smaller-scale or higherwavenumber components of motion. In the light of mean flow, this finding suggests that the existence of helicity in mean flow suppresses the energy cascade to fluctuating parts of motion. With this point in mind, we adopt $\Psi$ as a constraint on $\Phi$ and seek the state

$$
\Phi=\text { minimum under } \Psi=\text { constant, }
$$

with the aid of variational method. An Euler equation is given as

$$
\Omega=-\frac{1}{\lambda} \nabla \times \Omega \text {. }
$$

The solution of Eq. (4), which is regular at the centerline ( $r=0)$, is given by

$$
\Omega_{z}=\Omega_{z}^{(C)} J_{0}(\lambda r)
$$

where $J_{n}$ is the first-kind Bessel function of the $n$th order,

$$
\Omega_{z}^{(C)}=\Omega_{z}(0),
$$

and

$$
\Omega_{\theta}=-\Omega_{z}^{(C)} J_{1}(\lambda r) .
$$

From Eqs. (5) and (7), $\boldsymbol{U}$ has been expressed in terms of the Bessel function $J_{n}$ as

$$
U_{z}=U^{(N)}-\frac{1}{\lambda} \Omega_{z}^{(C)} J_{0}(\lambda r)
$$

and

$$
U_{\theta}=\frac{\Omega_{z}^{(C)}}{\lambda} J_{1}(\lambda r)
$$

where $U^{(N)}$ is the uniform axial velocity in a nonswirling pipe flow in the large Reynoldsnumber limit. The eigenvalue $\lambda$ is determined by the condition

$$
J_{1}\left(\lambda r_{M}\right)=0
$$

where $r_{M}$ is the effective radius of the wall. The first zero in Eq. (10) gives

$$
|\lambda| r_{M}=3.8
$$

Equation (8), together with Eq.(11), shows that the reversal of central axial velocity takes place if the helicity of swirling flow exceeds a criterion.

\section{Reference}

Akira Yoshizawa, Nobumitsu Yokoi, Shoiti

Nisizima, Sanae-I. Itoh, Kimitaka Itoh:

NIFS-680 (2001) 